\title{
The EUV Excess in Magnetic Cataclysmic Variables
}

\author{
JOHN K. WARREN ${ }^{1}$ AND KOJI MUKAI ${ }^{2}$ \\ ${ }^{1}$ Space Sciences Laboratory, University of California, Berkeley, CA 94720, USA \\ ${ }^{2}$ Code 668, NASA/Goddard Space Flight Center, Greenbelt, MD 20771, USA
}

We present preliminary analysis of $E U V E$ pointed data of 8 magnetic cataclysmic variables. Blackbody temperatures, luminosities, and interstellar columns have been better constrained. Using these luminosities we look for correlations between the EUV excess (over optical and hard $\mathrm{X}$-rays) and various system parameters. While it appears there is no correlation between the EUV excess and the system inclination and orbital period, correlations are suggested between the EUV excess and the longitude of the accretion spot, the colatitude of the accretion spot, the white dwarf magnetic field, and the magnetic capture radius.

\section{Introduction}

Magnetic cataclysmic variables, or polars, are cataclysmic variables (CVs) with a white dwarf magnetic field of $\sim 10-60 \mathrm{MG}$. They have no accretion disk and the accretion column lands directly on the white dwarf in a small region(s) near one or both of its magnetic poles. See Cropper (1990) for a review. In the standard (outdated) model the accreting material passes through a standoff shock just above the white dwarf, cooling via optically thin thermal bremsstrahlung hard X-rays (HXR) and optical gyrocyclotron radiation. Half of this radiation is reradiated by the white dwarf in the extreme ultraviolet (EUV) and soft X-rays (SXR). This model of magnetic CV accretion predicts that the ratio $R$ of observed EUV luminosity to the sum of the observed HXR and optical luminosities is obeys the simple relation $R \sim 0.5$, where

$$
R=\frac{L_{e u v}}{L_{h x r}+L_{o p t}}=\frac{L_{h x r, 0}\left(1-a_{x}\right)}{L_{h x r, 0}\left(1+a_{x}\right)+L_{o p t}} \sim \frac{1-a_{x}}{1+a_{x}}
$$

$L_{e u v}, L_{h x r}$, and $L_{\text {opt }}$ are the EUV, HXR and optical luminosities, respectively, $L_{h x r, 0}$ is the HXR luminosity directed toward the white dwarf, and $a_{x} \sim .3$ is the X-ray albedo of the white dwarf. The above approximation, in which the optical luminosity is small enough to be neglected, applies in many systems.

Since the days of $H E A O-1$ it has been known that $R$ far exceeds the expected value of 0.5 in many systems. This has been known historically as the SXR excess, but which we call here the EUV excess. Several theories have been proposed to explain the EUV excess, the most promising of which is direct mechanical heating of the white dwarf atmosphere by dense filaments in the accretion stream (Kuijpers \& Pringle (1982), Frank, King, \& Lasota (1988)). Some as yet unknown mechanism is necessary to separate the flow (at the threading region or perhaps the $\mathrm{L} 1$ point) into segments which will then be turned into dense filaments by being pinched by the converging magnetic field lines and stretched by white dwarf tidal forces. No extra pressure or heat is needed to create these segments.

The EUV characteristics of CVs have been difficult to determine, because of their distances $(\geq 65 \mathrm{pc}$ ) and the high attenuation (a factor of 0.16 at $100 \mathrm{pc}$ and a column of $5 \times 10^{19} \mathrm{~cm}^{-2}$ ) suffered by the EUV photons traversing these distances. In polars, these characteristics include the size, shape, and temperature of the accretion region(s), the number of such regions, and the intervening column. New data from $E U V E$ allow tighter 
TABLE 1. Log of Observations

\begin{tabular}{|c|c|c|c|}
\hline $\begin{array}{l}\text { Star } \\
\text { Name }\end{array}$ & $\begin{array}{l}\text { Start } \\
\text { Date }\end{array}$ & $\begin{array}{l}\text { End } \\
\text { Date }\end{array}$ & $\underset{\text { (ks) }}{\text { Eff. Exp. }}$ \\
\hline V834 CEN & 1993 May 28.1 & May 29.6 & 42.7 \\
\hline EF ERI & 1993 Sep. 5.6 & Sep. 9.7 & 120.7 \\
\hline UZ For & 1995 Jan. 15.9 & Jan. 19.7 & 120.8 \\
\hline AM HER & 1993 Sep. 23.7 & Sep. 28.5 & 143.8 \\
\hline VV PUP & 1993 Feb. 7.9 & Feb. 9.3 & 46.0 \\
\hline RE J1149+284 & 1993 Feb. 22.8 & Feb. 25.3 & 81.0 \\
\hline RE J1844-741 & 1994 Aug. 17.6 & Aug. 24.0 & 150.0 \\
\hline RX J1938-461 & 1992 July 8.2 & July 9.7 & 37.3 \\
\hline RX J1938-461 & 1993 Aug. 16.1 & Aug. 17.1 & 29.1 \\
\hline RX J1938-461 & 1993 Oct. 6.3 & Oct. 10.7 & 143.2 \\
\hline MR SER & 1993 June 1.2 & June 2.8 & 48.7 \\
\hline AN UMA & 1993 Feb. 27.9 & Feb. 1.2 & 40.1 \\
\hline EK UMA & 1994 Dec. 14.3 & Dec. 16.0 & 50.0 \\
\hline
\end{tabular}

constraints on these characteristics and enable tighter constraints on the EUV excess. In this work we search for correlations between the EUV excess as observed by EUVE (Bowyer \& Malina (1991)) and other missions, and system parameters. EUVE derived parameters are in boldface in the figures.

\section{Observations}

To date, 11 polars have been observed by EUVE in pointed observations: V834 Cen, EF Eri, UZ For, AM Her, VV Pup, RE J1149+284, RE J1844-741, RX J1938-461, MR Ser, AN UMa, and EK UMa. A summary of these observations is provided in Table 1. The data of RE J1844-741 are not publicly available, and two of the other 10-MR Ser and EK UMa-were undetected. The 8 remaining spectra were detected, as expected, only in the short wavelength channel (70-190 $\AA$ ); no flux was detected in the medium wavelength $(140-380 \AA)$ or long wavelength $(280-760 \AA)$ channels. Blackbody fits were performed on these spectra, yielding $T_{\text {euv }}, N_{H}, \& L_{\text {euv }}$, i.e., the blackbody temperature, the interstellar column, and the bolometrically corrected blackbody luminosity, respectively. These, along with values from other missions (see Ramsay et al. (1994)), are plotted in Figure 1.

\section{Results and Discussion}

In addition to the $E U V E$ observations, we made use of the values compiled by Ramsay et al. (1994) of the HXR and optical luminosities of these 8 systems, as well as the blackbody parameters and HXR and optical luminosities of several other magnetic CVs. Ratios $R$ of observed EUV luminosity to the sum of the (non-simultaneously) observed HXR and optical luminosities were computed, according to Equation 1.1. We then plotted these values of $R$ against various system parameters: orbital inclination $i$, period $P$, longitude $\psi$ of the accretion region, colatitude $\beta$ of the accretion region, white dwarf 

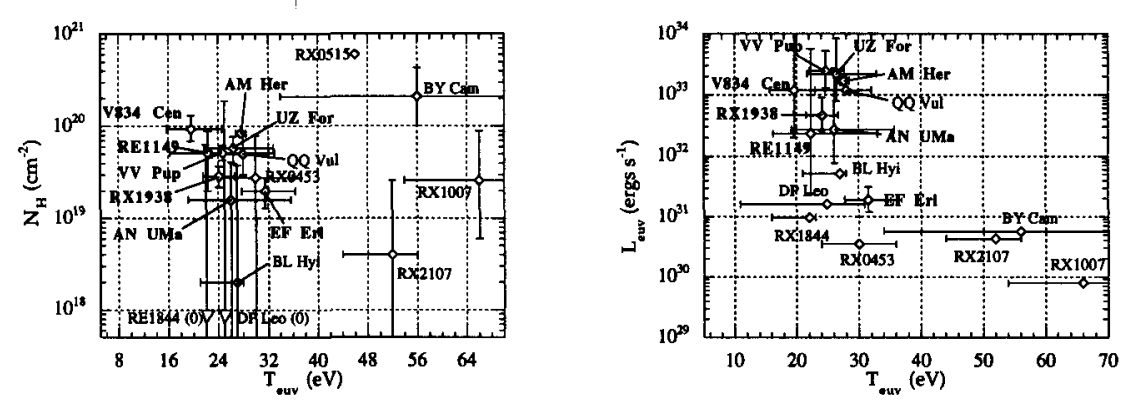

FigURE 1. Plots of EUV blackbody parameters $T_{\text {euv }}, N_{H}$, and $L_{e u v}$. EUV Results are in boldface.
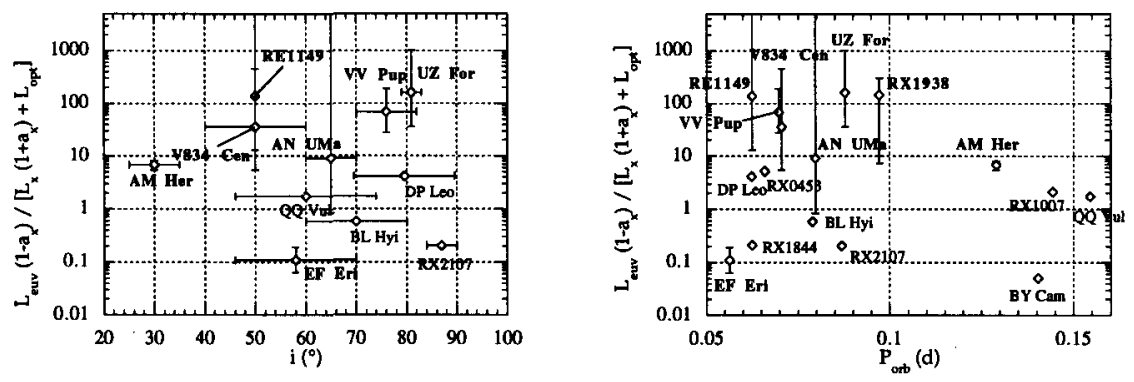

FIGURE 2. EUV excess vs. inclination $i$ and orbital period $P$.

magnetic field $B$, and magnetic capture radius $R_{c} . R_{c}$ is given by the equation of the ram pressure of the stream with the magnetic pressure (Mukai (1988)):

$$
R_{c}=1.48 \times 10^{10} B_{7}^{\frac{1}{11}} R_{9}^{\frac{12}{11}} D_{9}^{\frac{4}{11}} \dot{M}_{16}^{\frac{-2}{11}} M_{1}^{\frac{-1}{11}} \mathrm{~cm},
$$

where $B_{7}$ is the magnetic field in units of $10^{7}$ gauss, $R_{9}$ is the white dwarf radius in units of $10^{9} \mathrm{~cm}, D_{9}$ is the stream diameter in units of $10^{9} \mathrm{~cm}, \dot{M}_{16}$ is the mass transfer rate in units of $10^{16} \mathrm{gm} \mathrm{cm}^{-1}$, and $M_{1}$ is the mass of the white dwarf in units of $\mathrm{M}_{\odot}$.

We did not see a correlation between the EUV excess $R$ and the inclination $i$, indicating the absence of beaming effects. No correlation is observed between $R$ and the orbital period $P$, which is related to the secondary mass (Figure 2). However, there seems to exist a correlation between $R$ and the longitude $\psi$ of the accretion region, and between $R$ and the colatitude $\beta$ of the accretion region (Figure 3 ).

There may be a marginal correlation between $R$ and the magnetic field $B$ of the white dwarf. Associated with this there may also be a correlation between $R$ and the magnetic capture radius $R_{c}$ (Figure 4), indicating that in stars where the material is captured farther out the EUV excess is higher. The asynchronous system BY Cam is an exception in both plots. In any case, an anti-correlation between $R$ and $R_{c}$ is expected from the 

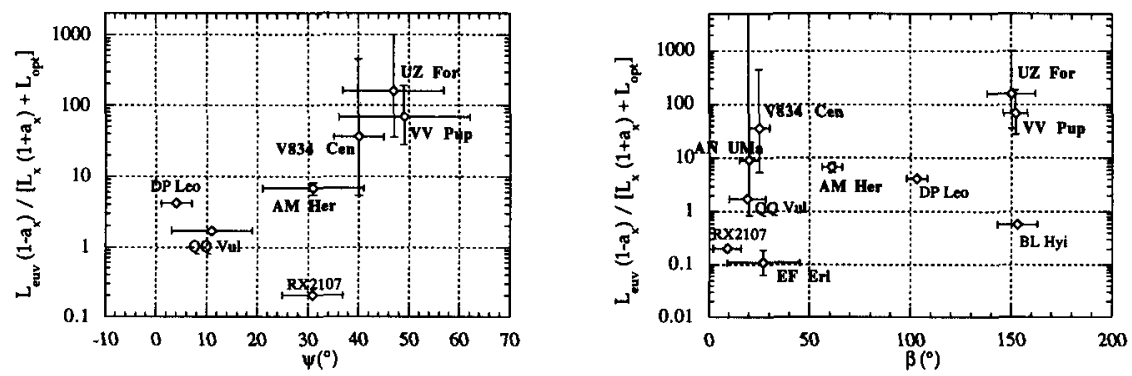

FIGURE 3. EUV excess vs. longitude $\psi$ and colatitude $\beta$ of accretion spot.
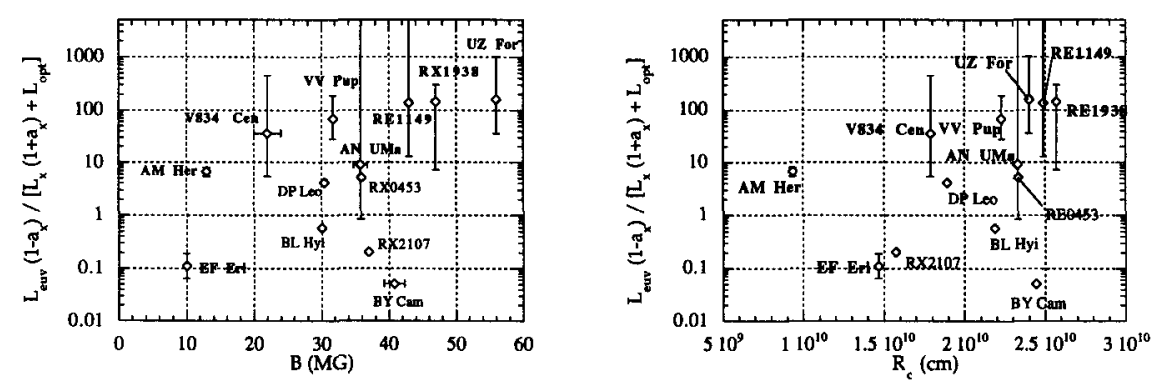

FIGURE 4. EUV Excess vs. white dwarf magnetic field $B$ and magnetic capture radius $R_{c}$.

theory that the Kelvin-Helmolz instability has more time to shred the filaments into fine droplets in streams that are captured farther out (Cropper (1990)). However, this anti-correlation is not observed (see also Ramsay et al. (1994)).

Further analysis is in progress, including the exploration of deviations from blackbody spectral shape in the brighter systems, the results of which will be the subject of a forthcoming paper.

This work was supported by NASA grant NAG 5-2641, and by NASA contract NAS5-32490.

\section{REFERENCES}

Bowyer, S. \& Malina, R. F. 1991, The EUVE Mission, in Extreme Ultraviolet Astronomy, ed. R. F. Malina \& S. Bowyer, New York: Pergamon Press, 397

Cropper, M. S. 1990, The Polars, Space Sci. Rev., 54, 195

Frank, J., King, A. R. \& Lasota, J. -P. 1988, The soft X-ray excess in accreting magnetic white dwarfs, A\&A, 193, 113 
KuiJPers, J. \& Pringle, J. E. 1982, Comments on radial white dwarf accretion, A\&AL, 114, L4-L6

MUKAI, K. 1988, Accretion streams in AM Her type systems., MNRAS, 232, 175

Ramsay, G., Mason, K. O., Cropper, M., Watson, M. G. \& Clayton, K. L. 1994, Rosat observations of AN UMa and MR Ser: the status of the soft X-ray excess in AM Her stars., MNRAS, 270, 692 

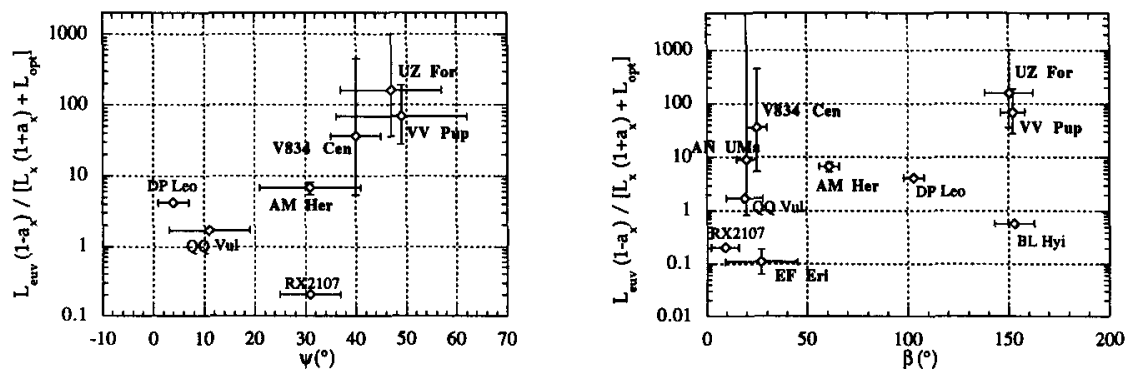

FIGURE 3. EUV excess vs. longitude $\psi$ and colatitude $\beta$ of accretion spot.
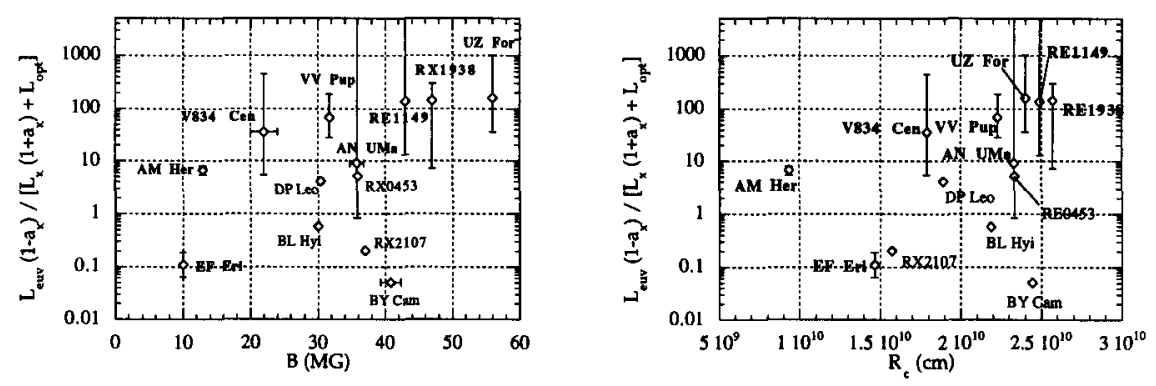

FIGURE 4. EUV Excess vs. white dwarf magnetic field $B$ and magnetic capture radius $R_{c}$.

theory that the Kelvin-Helmolz instability has more time to shred the filaments into fine droplets in streams that are captured farther out (Cropper (1990)). However, this anti-correlation is not observed (see also Ramsay et al. (1994)).

Further analysis is in progress, including the exploration of deviations from blackbody spectral shape in the brighter systems, the results of which will be the subject of a forthcoming paper.

This work was supported by NASA grant NAG 5-2641, and by NASA contract NAS5-32490.

\section{REFERENCES}

Bowyer, S. \& Malina, R. F. 1991, The EUVE Mission, in Extreme Ultraviolet Astronomy, ed. R. F. Malina \& S. Bowyer, New York: Pergamon Press, 397

Cropper, M. S. 1990, The Polars, Space Sci. Rev., 54, 195

Frank, J., KING, A. R. \& LAsota, J. -P. 1988, The soft X-ray excess in accreting magnetic white dwarfs, A\&A, 193, 113 
KuiJPers, J. \& Pringle, J. E. 1982, Comments on radial white dwarf accretion, A\&AL, 114, L4-L6

MUKAI, K. 1988, Accretion streams in AM Her type systems., MNRAS, 232, 175

Ramsay, G., Mason, K. O., Cropper, M., Watson, M. G. \& Clayton, K. L. 1994, Rosat observations of AN UMa and MR Ser: the status of the soft X-ray excess in AM Her stars., MNRAS, 270, 692 